\title{
The association of incidentally detected heart valve calcification with future cardiovascular events
}

\author{
Martijn J. A. Gondrie • Yolanda van der Graaf • \\ Peter C. Jacobs • Ay L. Oen - Willem P. Th. M. Mali • \\ On behalf of the PROVIDI Study Group
}

Received: 9 June 2010 /Revised: 23 September 2010 / Accepted: 25 September 2010 /Published online: 8 November 2010

(C) The Author(s) 2010. This article is published with open access at Springerlink.com

\begin{abstract}
Objectives This study aims to investigate the prognostic value of incidental aortic valve calcification (AVC), mitral valve calcification (MVC) and mitral annular calcification (MAC) for cardiovascular events and non-rheumatic valve disease in particular on routine diagnostic chest $\mathrm{CT}$.

Methods The study followed a case-cohort design. 10410 patients undergoing chest $\mathrm{CT}$ were followed for a median period of 17 months. Patients referred for cardiovascular disease were excluded. A random sample of 1285 subjects and the subjects who experienced an endpoint were graded for valve calcification by three reviewers. Cox-proportional hazard analysis was performed to evaluate the prognostic value.

Results 515 cardiovascular events were ascertained. Compared with patients with no valve calcification, patients with severe AVC, MVC or MAC had respectively 2.03 (1.482.78), 2.08 (1.04-4.19) and 1.53 (1.13-2.08) increased risks of experiencing an event during follow-up. For valve endpoints the hazard ratios were respectively 14.57 (5.1940.53), 8.78 (2.33-33.13) and 2.43 (1.18-4.98).

Conclusion Incidental heart valve calcification, detected on routine chest $\mathrm{CT}$ is an independent predictor of future
\end{abstract}

cardiovascular events. The study emphasises how incidental imaging findings can contribute to clinical care. It is a step in the process of composing an evidence-based approach in the reporting of incidental subclinical findings.

Keywords Survival analysis · Heart valves · Thorax · Cardiovascular diseases . Computed tomography

\author{
Abbreviations \\ AVC Aortic Valve Calcification \\ AVL Aortic Valve Leaflet \\ CHD Coronary Heart Disease \\ CI Confidence Interval \\ CT Computed Tomography \\ CVD Cardiovascular Disease \\ FOV Field of View \\ MAC Mitral Annular Calcification \\ MVC Mitral Valve Calcification \\ MVL Mitral Valve Leaflet \\ PAOD Peripheral Arterial Obstructive Disease \\ PROVIDI Prognostic Value of Unrequested Information \\ in Diagnostic Imaging
}

\section{Introduction}

Multidetector chest Computed Tomography (CT), which is usually performed during the diagnostic work-up of patients suffering from a variety of chest complaints, can yield extensive additional information about the heart, the lungs, the mediastinum, the vertebrae and the large vessels [1]. In the last decade, the image quality of chest Computed Tomography has improved and its usage has thereby increased considerably. A side effect of this intense usage is the frequent occurrence of incidental findings [2-4]. We
A. L. Oen

Department of Radiology, Elkerliek Hospital,

Helmond, Netherlands
M. J. A. Gondrie · P. C. Jacobs • W. P. T. M. Mali

Department of Radiology, University Medical Center Utrecht, Utrecht, Netherlands

M. J. A. Gondrie $(\bowtie) \cdot$ Y. van der Graaf

University Medical Center Utrecht,

Netherlands

(1) 
define these as imaging characteristics that are incidentally detected and unrelated to the clinical indication leading to the CT referral. Only follow-up studies can determine whether patients can benefit from these incidental findings as such studies uncover which incidental imaging abnormalities have or do not have clinically relevant prognostic value. The latter will serve to reduce redundant additional diagnostic work-up.

Examples of frequently encountered incidental findings are aortic valve calcification (AVC), mitral valve calcification (MVC) and mitral annulus calcification (MAC). These imaging characteristics are considered to be expression of the process of atherosclerosis [5-7] and have repeatedly been shown to be related to cardiovascular disease (CVD), cardiovascular death and even all-cause mortality [8-10]. However, in previous literature those image characteristics were mainly scored on transthoracic or transoesophageal echocardiography and to date no follow-up studies are available that have investigated the prognostic value of these aortic abnormalities when incidentally detected on routine chest $\mathrm{CT}$ in a clinical care population. Investigating this can provide an additional means of identifying those patients who can benefit from timely preventative measures [11].

The PROgnostic Value of incidental Information in Diagnostic Imaging (PROVIDI) Study is the first longitudinal study to investigate the prevalence and clinical relevance of incidental, subclinical image characteristics in a cohort consisting of patients with diagnostic chest CT. In this particular study we aimed to examine whether subclinical calcification of the aortic and mitral valve, detected incidentally on routine diagnostic chest $\mathrm{CT}$, can be used by radiologists as independent predictors of the occurrence of CVD and non-rheumatic valve disease in particular. Additionally, we examine whether incidentally detected calcified heart valves have prognostic value beyond the hitherto typically investigated CVD markers: aortic- and/or coronary arteries [12-16]. This study operates from radiologist's perspective and only seeks to incorporate information that is readily available in routine radiological setting. No allowance was made for the collection of additional clinical information, such as diabetes and renal failure, which may not routinely be available in such settings.

\section{Materials and methods}

\section{PROVIDI}

The PROVIDI study employs a case-cohort design [17], in cooperation with 8 participating hospitals in the Netherlands.
All patients aged 40 years and older who were referred to one of the participating hospitals with an indication for a chest CT between 2002 and 2005 were included $(N=$ 23,443). Patients diagnosed with primary lung cancer (including mesothelioma) or distant metastatic disease from other types of cancer (excluding haematological malignancies) were excluded, as it is highly unlikely that detection of incidental imaging characteristics will alter clinical decisionmaking in patients with a very poor prognosis. This resulted in a cohort of 14,366 patients. The PROVIDI study is approved by the Medical Ethics Committee of the Medical Ethics Committee of the University Medical Center Utrecht, as well as the ethical review boards of all other participating centres. Written informed consent was waived for all patients because of the retrospective design of this study. A privacy protocol was implemented to protect patient's anonymity.

\section{Study subjects and CT protocols}

In this study patients with a $\mathrm{CT}$ indication directly related to suspected cardiovascular disease were excluded $(n=2303)$ from the PROVIDI cohort in order to ensure that valve calcification observed was truly incidental. Additionally, a total of 1653 patients from a randomly chosen hospital were excluded, to serve as an external validation sample for future research conducted within PROVIDI. The resulting cohort consisted of 10,410 patients. Consistent with a casecohort design a random sample - called subcohort-was taken from this cohort immediately after the patients who experienced an outcome event were ascertained to serve as a control. It was sampled so that the resulting sample would be at least $10 \%$ [18] of the full cohort and twice as large as the specific group of outcome event under study, with a reasonable margin for error (subcohort, $n=1285$ ). Figure 1 shows a flowchart of the study design and selection of the study population.

When study subjects had more than one Chest CT examination during follow-up, only the first Chest $\mathrm{CT}$ was used. Chest CTs were obtained with 2-, 4-, 8-, 16-, 32- or 64-slice systems from different vendors. All types of contrast enhanced CT protocols were considered eligible as long as the field-of-view (FOV) included the heart and the full length of the thoracic aorta. Slice thickness varied according to the Chest $\mathrm{CT}$ indication and corresponding protocol and-due to exclusion of CT examinations performed for a cardiovascular indication-none of the CT studies were ECG gated. Patient characteristics, information on slice thickness and the use of a contrast agent were abstracted from Chest CT reports by a research physician, who also assessed the diagnostic Chest CT indication and classified them as 'pulmonary disease', 'haematological malignancy', 'mediastinal disease', 'ruled- 


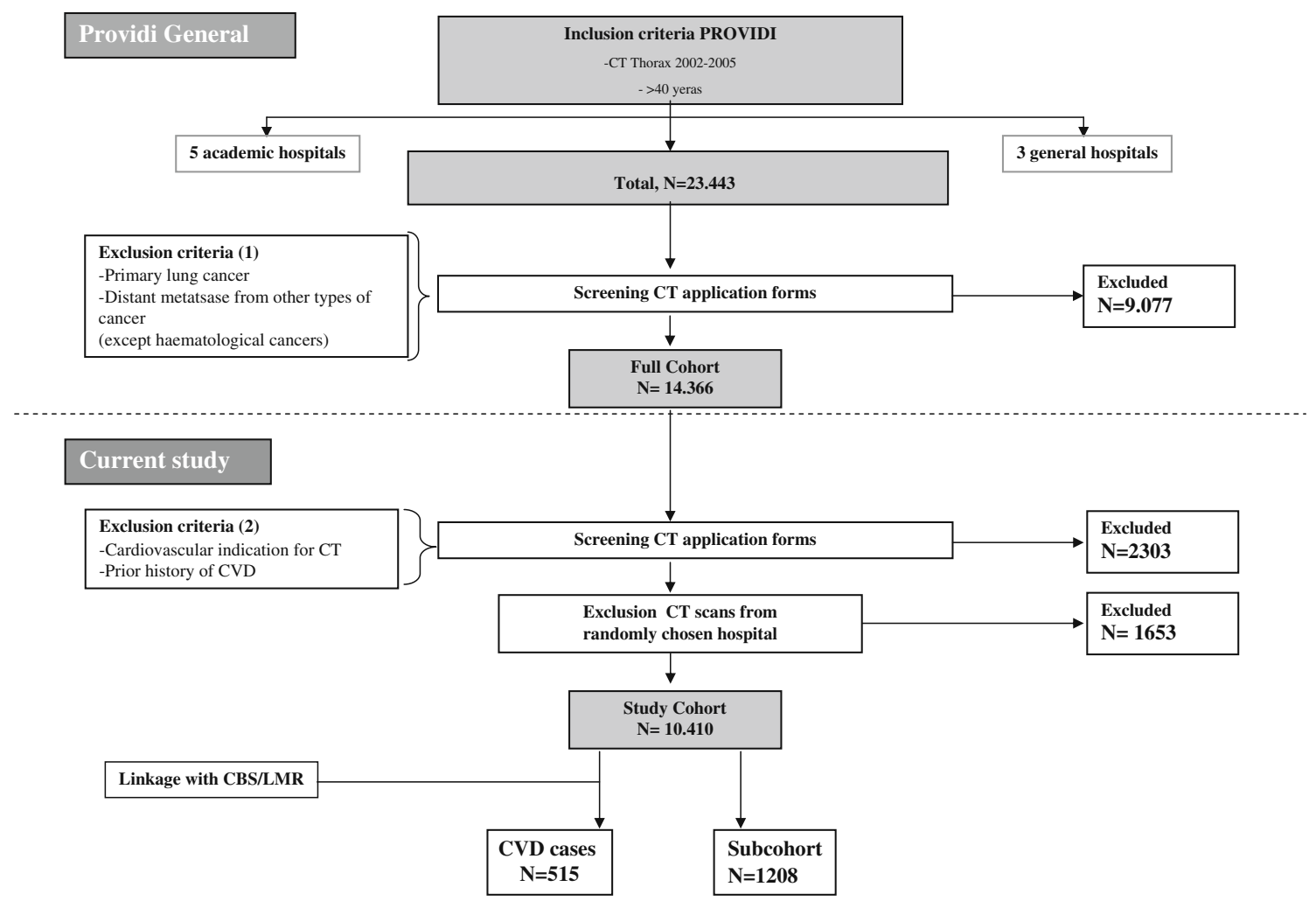

Fig. 1 Flowchart of study design. CBS: Dutch central bureau of statistics. LMR: Dutch medical data registration

out pulmonary malignancy', 'pulmonary embolism' or 'other CT indications'.

\section{Image characteristics}

The CT-findings were scored for valve calcification by three readers: one board certified radiologist with 10 years' experience and two research physicians with two and three years' Chest CT experience respectively. All readers were blinded for patient characteristics and outcome status. CT images were stored in DICOM format and read using a commercially available software program (Dicomworks, version 1.3.5). All CTs were analysed in the axial plane; no other reformatted planes were included for analysis. Readings were performed using a standardised score form including information on the type of CT protocol used and the use of contrast agent and imaging quality. This was defined as good: clearly visible, minimal movement artefacts, adequate: Slight blurring and/or movement artefacts, or poor: major defects significantly affecting visibility. These classes reflect the reliability with which the scoring of CT characteristics was possible.

Calcification of the heart valves was anatomically subdivided into calcification of the aortic valve leaflets (AVL), the mitral valve leaflets (MVL) and the mitral annulus. MVL calcification present as linear calcification between the area of the left atrium and ventricle. Mitral Annulus Calcification (0, absent; 1, present) are visible as round or curled spots at the periphery of this horizontal leaflet plane. AVL and MVL calcification was graded as: grade 0, absent; grade 1, mild (one leaflet affected); grade 2 , severe ( 2 or 3 leaflets affected), comparable to other studies [19, 20].

Calcification was also visually assessed in the coronary arteries and the thoracic aorta, as premature calcification in these anatomical regions has already frequently been related to CVD in the literature. Calcification of the aorta was also assessed in the ascending and descending part and in the supra-aortic arteries branching from the aortic arch. An overall aorta score was compiled and graded as absent, mild, moderately, or severe affected aorta. Calcification in the coronaries was scored in a similar fashion. Both aortic and coronary visual grading were partly comparable to a previously described grading of coronary calcification [21]. Tables 1 and 2 provide information about the visual grading and examples of image characteristics are presented in Fig. 2.

Scoring was trained under the supervision of an experienced board certified chest radiologist. The reproducibility of visual grading was successfully evaluated in a previous study [22]. Briefly, weighted kappa 0.78, 0.58, 
Table 1 Definitions used for visual grading of valve calcification on routine diagnostic Chest CT

\begin{tabular}{llll}
\hline & Grades & \\
\cline { 2 - 4 } Finding & 0 & 1 & 2 \\
\hline Aortic valve calcification & none & $\begin{array}{c}\text { One small calcification or linear calcification } \\
\text { of one leaflet }\end{array}$ & Linear calcification on two or three leaflets \\
Mitral valve calcification & none & $\begin{array}{c}\text { One small calcification or linear calcification } \\
\text { of one leaflet }\end{array}$ & Linear calcification on two leaflets \\
\hline
\end{tabular}

0.56 and $0.87,0.56$, and 0.70 for inter- and intra observer agreement of AVC, MVC and MAC. The weighted kappa's for inter- and intraobserver agreement were $0.77,0.91$ for the coronaries, $0.72,0.88$ for the aorta and $0.89,0.96$ for the supra aortic calcification respectively.

\section{Follow-up and outcomes}

We recorded incident fatal and non-fatal CVD events for a mean of 17 months (max. 48 months). Endpoint status was obtained through linkage of patients with the National Death Registry and the National Registry of Hospital Discharge Diagnoses from January 2002 to December 2005. Database linkage was performed with a validated probabilistic method [23-26]. In these databases, cause of death and the indications for hospitalisation are coded according to the International Classification of Diseases, $9^{\text {th }}$ and $10^{\text {th }}$ revision (ICD-9, ICD-10) [27]. Correct designation of causes of death has been established within a comparison study with patient medical records [28].

A trained research physician abstracted all valid endpoints from these databases on the basis of ICD codes. Fatal and non-fatal CVD events with an atherosclerotic background were defined by ICD-9 codes as coronary heart disease (CHD) (codes 410-414), heart failure (code 428), peripheral arterial disease (PAOD) (codes 440, 443-444), aortic aneurysm (code 441), cerebrovascular disease (codes 430-438), non-rheumatic valve disease (code 424), or other forms of heart disease (e.g. cardiac dysrythmias, or cardiomyopathy $(425,427)$ ). Only the latter (endpoints with codes 424) were used in the analysis for fatal and nonfatal non-rheumatic valve endpoints. In case of multiple valid endpoints in the same patient, cause of death prevailed over hospital admissions or else the first hospital discharge diagnosis was used.

\section{Data analysis}

Descriptive information of patients, imaging characteristics, CT indications and follow-up time were evaluated for patients from the subcohort (random sample from cohort) and patients with cardiovascular disease (cases). We used regression methods implemented in SPSS software (SPSS 14.0, Chicago, IL, USA) to impute missing values $(<3 \%$ for the scored imaging characteristics). All other analyses were performed with R software, version 6.2.

Cox proportional hazard analysis was used to investigate whether valve calcification can be used as a predictor of CVD events and non-rheumatic valve disease in particular. Apart from crude associations, the hazard ratios were also adjusted for age, sex, indication for $\mathrm{CT}$ and type of medical hospital in which the $\mathrm{CT}$ was performed (tertiary/secondary).

Table 2 Definitions used for visual grading of coronary and aortic calcification on routine diagnostic Chest CT

\begin{tabular}{|c|c|c|c|c|}
\hline \multirow[b]{2}{*}{ Finding } & \multicolumn{4}{|l|}{ Grades } \\
\hline & Absent & Mild & Moderate & Severe \\
\hline $\begin{array}{l}\text { Coronary calcification } \\
(\mathrm{LM}, \mathrm{LAD}, \mathrm{LCX}, \mathrm{RCA})^{\mathrm{a}}\end{array}$ & none & $\begin{array}{l}\leq 5 \text { calcified foci or } 1 \\
\text { calcification extending } \\
\text { over } \geq 2 \text { slices }\end{array}$ & $\begin{array}{l}>5 \text { calcified foci or } 2 \\
\text { calcification extending } \\
\text { over } \geq 2 \text { slices }\end{array}$ & $\begin{array}{l}>6 \text { calcified foci and } \geq 3 \\
\text { calcification extending } \\
\text { over } \geq 2 \text { slices }\end{array}$ \\
\hline $\begin{array}{l}\text { Aortic and supra aortic } \\
\text { calcification }\end{array}$ & none & $\begin{array}{l}\leq 4 \text { calcified foci or } 1 \\
\text { calcification extending } \\
\text { over } \geq 3 \text { slices }\end{array}$ & $\begin{array}{l}>4 \text { calcified foci or } 2 \\
\text { calcification extending } \\
\text { over } \geq 3 \text { slices }\end{array}$ & $\begin{array}{l}\text { calcified aorta covering } \\
\text { multiple segments }\end{array}$ \\
\hline
\end{tabular}

Note that this table needs to be interpreted as a guideline. Clinical experience of a radiologist is essential to assign points

${ }^{a} L M$ left main; $L A D$ left anterior descending; $L C X$ left circumflex; $R C A$ right coronary artery 

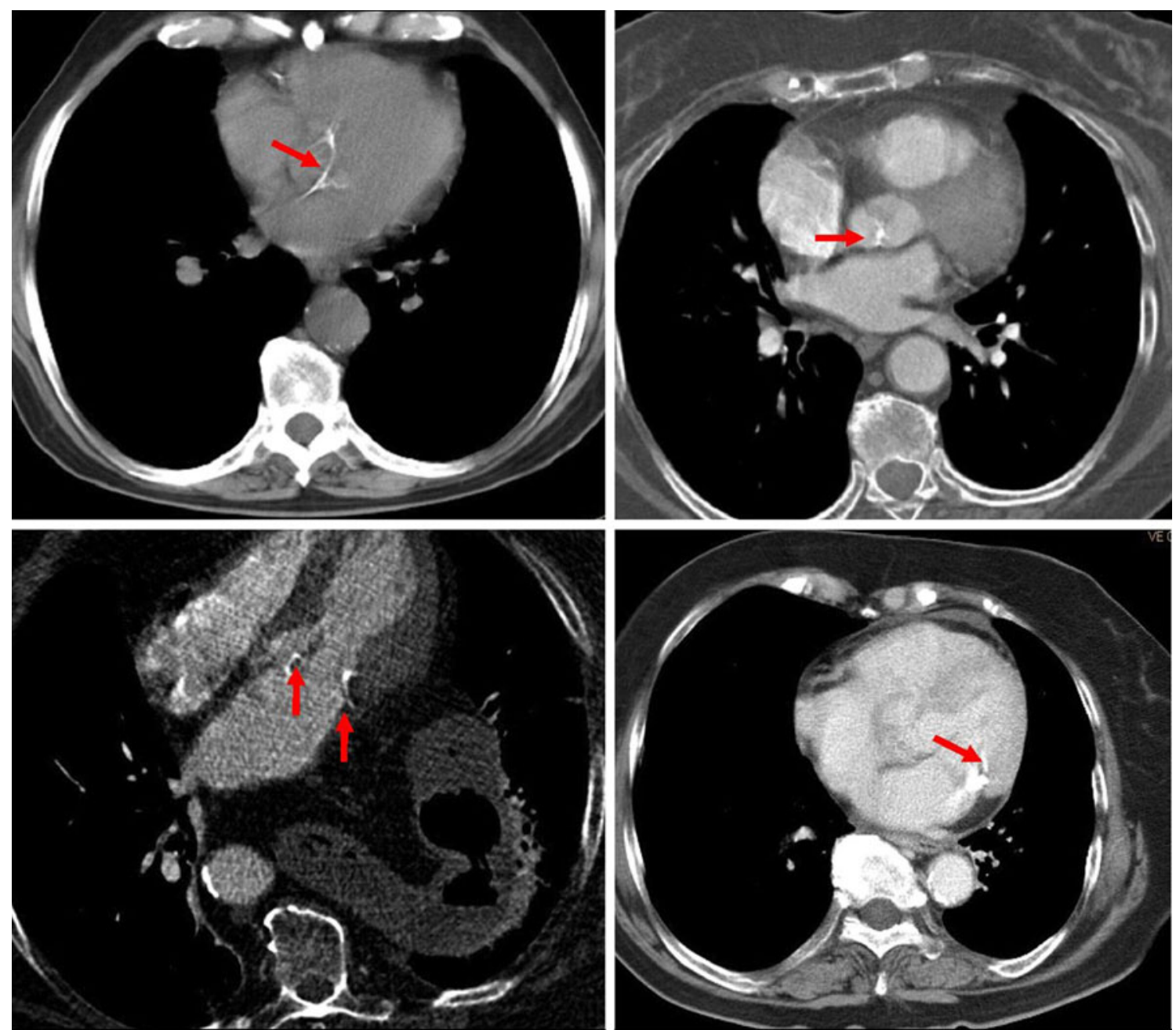

Fig. 2 Image examples. Upper left image shows two affected leaflets of the aortic valve. The upper right shows one calcified leaflet of the aortic valve. The lower left image shows annular calcification of the mitral valve and the lower right image shows an affected leaflet of the mitral valve

Interaction terms were included in the model to test whether imaging characteristics (valve calcification• section thickness (continuous), calcification • use of contrast agent (yes/no), calcification $\cdot$ imaging quality (bad, moderate, good), calcification - age (continuous) and calcification gender (male/ female)) modified the associations. Furthermore, survival curves of the strongest predictors were presented to visually illustrate the effect of the adjusted hazard ratios on the occurrence of CVD. The curves show the observed proportion of patients being free of CVD events during follow-up per stratum of the heart valve score. Finally, a sub-analysis was performed to investigate whether the predictive value of calcified heart valves for future CVD events attenuated when additional commonly used atherosclerotic markers were included in the model. In this analysis, the adjusted hazard ratios were additionally corrected for calcification in aorta and/or coronary arteries.
All hazard ratios were modified based on robust variance estimates. These adaptations were carried out with the method according to Prentice in which all subcohort members are equally weighted [17]. This method has been shown to resemble most closely estimates from a fullcohort analysis [18].

\section{Results}

A total of 121 subjects (44 out of 559 cases (7.9\%), and 77 out of 1285 subjects from the subcohort $(6.0 \%)$ ) were excluded from analyses because CTs could not be retrieved from hospital CT databases. Table 3 presents descriptive information on patient and imaging characteristics: The mean age of the patients from the subcohort was 61.5 years and $58 \%$ were male. The overall prevalence in the 
Table 3 Baseline characteristics

\begin{tabular}{|c|c|c|}
\hline General information & $\operatorname{Subcohort}(n=1208)$ & CVD $\operatorname{cases}(\mathrm{n}=515)$ \\
\hline Male, $[\%]$ & 58 & 65 \\
\hline Age, y $[S D]$ & $61.5(40-96)$ & $67.9(40-96)$ \\
\hline Follow-up, days [SD] & $620(1-1460)$ & $322(1-1369)$ \\
\hline Academic referral centre, $[\%]$ & 77 & 77 \\
\hline Use of contrast agent [\%] & 68 & 67 \\
\hline \multicolumn{3}{|l|}{ Image quality, [\%] } \\
\hline Good & 72 & 74 \\
\hline Moderate & 24 & 24 \\
\hline Bad & 3 & 2 \\
\hline \multicolumn{3}{|l|}{ Indication category, $[\%]$} \\
\hline (1)Lung disease & 37 & 47 \\
\hline (3)Haematological malignancies & 11 & 6 \\
\hline (4)Mediastinal abnormalities & 11 & 8 \\
\hline (5)Ruled-out pulmonary malignancy ${ }^{\mathrm{a}}$ & 24 & 21 \\
\hline (6)Pulmonary emboli & 6 & 8 \\
\hline (8)Other indications & 11 & 10 \\
\hline \multicolumn{3}{|l|}{ Thoracic aorta calcification } \\
\hline None & 39 & 18 \\
\hline Mild & 30 & 25 \\
\hline Moderate & 19 & 33 \\
\hline Severe & 12 & 24 \\
\hline \multicolumn{3}{|l|}{ Coronary arteries calcification } \\
\hline None & 33 & 12 \\
\hline Mild & 24 & 22 \\
\hline Moderate & 25 & 29 \\
\hline Severe & 18 & 37 \\
\hline \multicolumn{3}{|l|}{ Aortic valve calcification, [\%] } \\
\hline None & 72 & 53 \\
\hline Calcification on one leaflet & 16 & 20 \\
\hline Calcification on two or three leaflets & 11 & 27 \\
\hline \multicolumn{3}{|l|}{ Mitral valve calcification, [\%] } \\
\hline None & 91 & 82 \\
\hline Calcification on one leaflet & 7 & 14 \\
\hline Calcification on two leaflets & 2 & 4 \\
\hline Presence of annular calcification mitral valve [\%] & 12 & 20 \\
\hline
\end{tabular}

a Pulmonary mass, which is proven
proportions
are means [SD] or

a Pulmonary mass, which is proven
proportions
are means [SD] or

a Pulmonary mass, which is proven
proportions
are means [SD] or

a Pulmonary mass, which is proven
proportions
are means [SD] or

subcohort patients of aortic valve, mitral valve and mitral annular calcification was respectively $27 \%, 9 \%$ and $12 \%$. The proportion of moderately or severely affected coronary arteries was $43 \%$ and $31 \%$ for the aorta in subcohort patients. A total of 515 patients experienced a cardiovascular event during follow-up (for specification according ICD codes see Appendix). With regard to the cases the proportions of calcified heart valves, coronary arteries and aorta were all higher. Cases were also older (mean: 68 years) and were more often male $(65 \%)$.

Table 4 shows that the risk of a CVD event increased with an increase in the stratum of valve calcification. After adjustment for age, sex, CT indication and type of referral centre the hazard ratio for 1 affected aortic valve leaflet was no longer significant (HR 1.18, 95\% CI 0.87-1.61). However, with two or three aortic leaflets affected, the risk doubled (HR 2.03, 95\% CI 1.48-2.78). When one leaflet of the mitral valve was affected, the risk increased 1.83 -fold (95\% CI 1.26-2.64) and 2.08-fold (95\% CI 1.04-4.19) when two mitral valve leaflets were affected. Annular calcification increased the risk during follow-up with 53\% (HR 1.53, 95\% CI 1.13-2.08). Figures 3 and 4 visually present the prognostic effect of aortic and mitral valve calcification adjusted for age, sex, imaging indication and 
Table 4 Risk of any CVD event $(n=515)$ associated with increasing calcified valve score in clinical care patients undergoing routine diagnostic chest CT

CVD cardiovascular disease. $C I$ confidence interval

a Adjusted model: hazard ratio's adjusted for age, sex, imaging indication, referral centre
Hazard ratios $(95 \% \mathrm{CI})$

Crude

Adjusted model ${ }^{\mathrm{a}}$

Aortic valve calcification

None

1

$1.79(1.36-2.36)$

1

One affected leaflet

Two or three affected leaflets

$3.32(1.83-4.96)$

$1.18(0.87-1.61)$

$2.03(1.48-2.78)$

Mitral valve calcification

None

1

$2.66(1.89-3.75)$

1

One affected leaflet

$3.46(1.82-6.37)$

$1.83(1.26-2.64)$

$2.08(1.04-4.19)$

Mitral annular calcification

None

1

Presence

$2.11(1.59-2.81)$
$1.53(1.13-2.08)$ referral centre. The proportion of patients with a severely calcified aorta or mitral valve who suffered a CVD event within 3 years was $5.5 \%$ and $7 \%$, respectively. Linearly extrapolated to more widely used 10 -year risk predictions, these proportions would be $18 \%$ and $23 \%$. No statistically significant interactions were found between the valve and annular scores and section thickness (range $p=0.27-0.30$ ), the use of intravenous contrast medium (range $p=0.13$ 0.27 ), imaging quality (range $p=0.11-0.92$ ), age (range $p=$ $0.13-0.69$ ) or gender(range $p=0.42-0.90$ ).

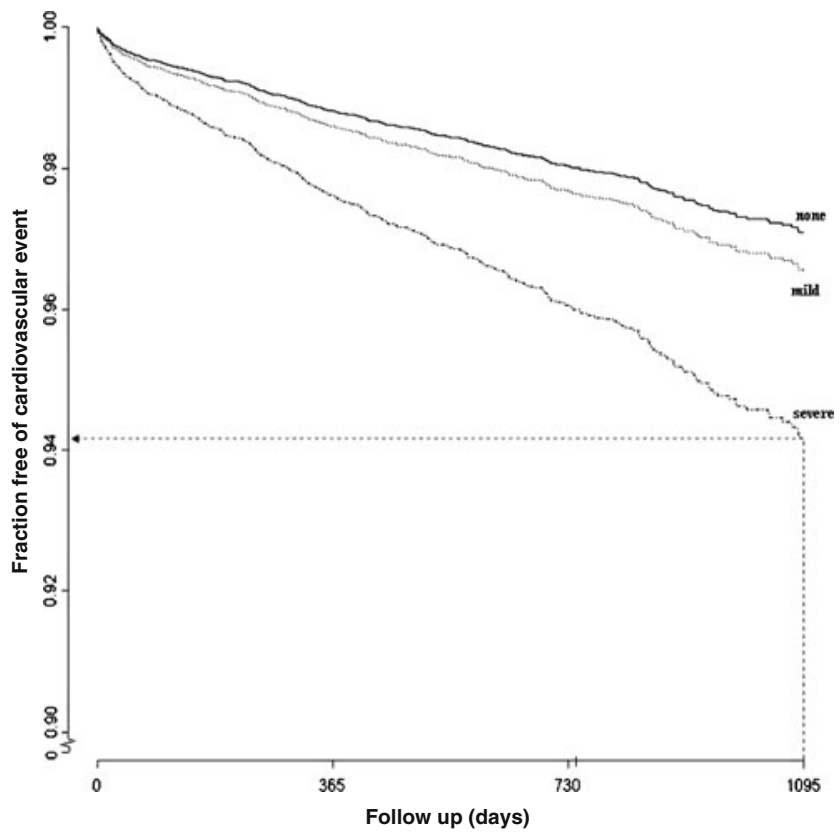

Fig. 3 Proportion of patients free of cardiovascular events according the aortic valve score adjusted for age, sex, imaging indication, referral centre. The dotted line is included as an example and indicates the proportion of severly affected patients free of cardiovascular events after 3 years of follow-up
Looking specifically at the relationship between incidentally detected valve calcification and the occurrence of non-rheumatic valve disease $(n=43)$ we could demonstrate strongly increased hazard ratios, yet the pattern of the associations was the same (Table 5). For a moderately affected aortic valve the risk increased 2.40 -fold $(95 \% \mathrm{CI}$ $0.78-7.53)$. When the aortic valve was severely affected the risk increased 14.57 -fold (95\% CI 5.19-40.53). The

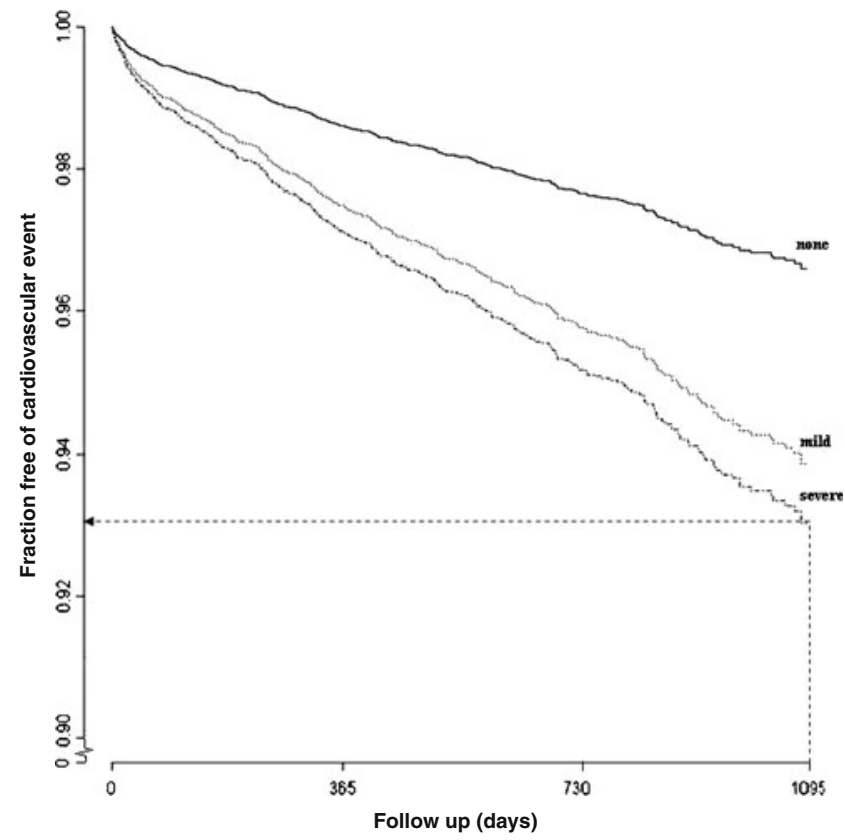

Fig. 4 Proportion of patients free of cardiovascular event according the mitral valve score adjusted for age, sex, imaging indication, referral centre. The dotted line is included as an example and indicates the proportion of severely affected patients free of cardiovascular events after 3 years' follow-up 
Table 5 Risk of non-rheumatic valve disease $(n=43)$ associated with increasing calcified valve score in clinical care patients undergoing routine diagnostic chest $\mathrm{CT}$
CVD cardiovascular disease. $C I$ confidence interval

${ }^{a}$ Adjusted model: hazard ratios adjusted for age, sex, imaging indication, referral centre

\begin{tabular}{|c|c|c|}
\hline & \multicolumn{2}{|c|}{ Hazard ratios $(95 \% \mathrm{CI})$} \\
\hline & Crude & Adjusted model $^{\mathrm{a}}$ \\
\hline \multicolumn{3}{|l|}{ Aortic valve calcification } \\
\hline None & 1 & 1 \\
\hline One affected leaflet & $2.62(0.96-7.13)$ & $2.40(0.78-7.53)$ \\
\hline Two or three affected leaflets & $16.27(7.85-33.69)$ & $14.57(5.19-40.53)$ \\
\hline \multicolumn{3}{|l|}{ Mitral valve calcification } \\
\hline None & 1 & 1 \\
\hline One affected leaflet & $5.17(2.42-11.0)$ & $3.84(1.77-8.34)$ \\
\hline Two affected leaflets & $11.27(4.02-34.40)$ & $8.78(2.33-33.13)$ \\
\hline \multicolumn{3}{|l|}{ Mitral annular calcification } \\
\hline None & 1 & 1 \\
\hline Presence & $3.15(1.62-6.51)$ & $2.43(1.18-4.98)$ \\
\hline
\end{tabular}

corresponding hazard ratios for the mitral valve were respectively $3.84(95 \%$ CI $1.77-8.34)$ and 8.78 (95\% CI 2.33-33.3). The presence of mitral annular calcification increased the risk with a factor 2.43 (95\% CI 1.18-4.98). Note however that for lack of power, these associations were not visually presented or further adjusted in the prospective sub-analysis.

\section{Sub-analysis}

When coronary and aortic calcification were included in the models for any CVD event, the hazard ratio for a moderately affected aortic valve decreased to 0.96 (95\% CI $0.70-1.32$ ) and for a severely affected aortic valve it decreased to 1.46 (95\% CI 1.04-2.06). For the mitral valve these ratios became 1.57 (95\% CI 1.08-2.28) and 1.79 (95\% CI 0.85-3.76), respectively. The corrected hazard ratio for mitral annular calcification was 1.19 (95\%CI 0.86-1.66).

\section{Discussion}

In this study, we examined whether calcification of the aortic valve, mitral valve and mitral annulus, incidentally detected on routine diagnostic chest CTs, could be used as predictors for CVD events and non-rheumatic valve abnormalities in particular. Furthermore we investigated whether these image characteristics remained prognostically relevant when they were modelled with commonly used predictive coronary and aortic calcification $[14-16,29,30]$. We found that, within a $95 \%$ confidence range, valve and annular calcification were independent predictors of CVD events. Considering the anatomical basis, the strongest associations were found for future valve abnormalities. A sub-analysis showed that these image characteristics only partly lost their prognostic capacity for CVD when jointly modelled with coronary and aortic calcification. Imaging parameters (slice thickness, use of intravenous contrast agents, or quality of the CT images), age or sex did not significantly modify the associations found for valve calcification in relation to future CVD events. This indicates that additional scoring of valve and annular calcification could provide a valuable contribution to the prediction of CVD events with hitherto commonly used atherosclerotic image characteristics on chest CT.

Millions of diagnostic CT examinations are made every year and their usage is still increasing because of the evolving quality of the CT images and the aging population [31]. An inevitable side-effect of this process is the increased number of incidentally detected subclinical image characteristics. Using this information for prognostic purposes is attractive as it can be freely abstracted from existing imaging data and comes with no extra exposure to ionising radiation. However, longitudinal studies, such as this one, are needed to expose the natural course of these findings and to demonstrate any (or lack of) relationship with future events. Ultimately, such studies can help in composing an evidence based approach to reporting incidental subclinical findings.

The prevalence of valve calcification in our study was $27 \%$ for AVC, 9\% for MVC and 12\% for MAC. These numbers are comparable to other studies, where the prevalence was reported to be $13-31 \%[9,10,19]$ for AVC, $9 \%$ for MVC[20] and 6-14\% for MAC [6, 9, 10]. In this study we also demonstrated a relationship between the investigated image characteristics and CVD events. Compared with other studies, which were mainly conducted in asymptomatic elderly populations, our study 
demonstrates these associations in a clinical care population. Another difference is that we scored incidental valve calcification on a very heterogeneous set of routinely made diagnostic chest CTs, instead of the transthoracic or transoesophageal echocardiography mainly used in other studies. This study demonstrates how incidentally detected CT image characteristics (simply scored with minimal extra reviewing time) can be used in daily clinical care for prognostic purposes. The results provide an additional means of improving the identification of high-risk patients, who can benefit from timely preventative measures [11]

Table 4 shows that ancillary valve calcification is an independent predictor of CVD events after correction for predictors readily available to radiologists. These results are in keeping with previous literature investigating aortic valve calcifications[32] and provide practical benefits compared with hitherto widely used atherosclerotic imaging characteristics (e.g. aortic and coronary calcification) as valve calcification can be scored very simply and quickly. However we would also like to stress that one image characteristic does not have to exclude another in the prediction of CVD: the sub-analysis showed that the prognostic effect of ancillary valve calcification did not attenuate completely when jointly modelled with coronary and aortic calcification. This could mean that predictions of CVD improve (in terms of accuracy) when those image characteristics are incorporated into one prediction model for CVD events. Note however that future research including other statistical model performance tests should confirm this.

This study has also some potential limitations. No clinical information from patients' medical records was available, which could have influenced the exclusion of patients already known with cardiovascular disease as we could only exclude patients based on their CT report. However, we excluded approximately $16 \%$ of patients from the baseline cohort based on information from CT reports and this is largely in line with the overall prevalence of symptomatic CVD in the general US population of whites (11.2\%) [33], indicating that this was unlikely to have affected our study population significantly. Clinical information from medical records might also be used to further adjust the hazard ratios: it is known that chronic conditions such as diabetes, renal dysfunction and metabolic syndrome [34-37]affect the incidence of both CVD and valve calcifications. Despite its potential effect[38], we did not seek to collect data on ethnicity. However, as this research is aimed at routine radiological care, where information about traditional risk factors is often unavailable, these omissions are representative of routine radiological practice. The additive nature of the 'radiological red flags' that may result from the clinical implementation of lessons learnt from this research may cause the identification of atrisk patients whom have already been identified by referring clinicians as being at risk. Such double identification of at risk patients on the basis of gratis (without extra radiation exposure) radiological information would not have any negative consequence for patients themselves and might serve as reinforcement for referring clinicians in cases where patients had already received preventative care (e.g. referring clinicians may verify whether the 'redflagged' patient is receiving optimal preventative care in such instances).

\section{Conclusion}

The PROVIDI study provides a new approach to employing freely available and potentially valuable prognostic information extrapolated from routine diagnostic imaging. This particular study showed that ancillary AVC, MVC and MAC, detected on routine care, diagnostic chest CT in a clinical care population, can be used by radiologists to predict cardiovascular events. Ultimately, prognostic information can be implemented in $\mathrm{CT}$ reports. Note that the impact of providing this information on risk stratification and following preventative measures should be investigated in another study.

Acknowledgements The PROVIDI Study Group consists of: J. Laméris (Dept. of Radiology, Academic Medical Center, Amsterdam), C. van Kuijk (Dept. of Radiology, VU University Medical Center Amsterdam), W. ten Hove (Dept. of Radiology, Gelre Hospitals, Apeldoorn), M. Oudkerk (Dept. of Radiology, University Medical Center Groningen), Ay L. Oen(Dept. of Radiology, Elkerliek Hospital, Helmond), J. Wildberger (Dept. of Radiology, Academic Hospital Maastricht), J. van Heesewijk (Dept. of Radiology, St. Antonius Hospital, Nieuwegein), W. Mali (Dept. of Radiology, University Medical Center Utrecht) and Y. van der Graaf (Julius Center for Health Sciences and Primary Care, University Medical Center Utrecht).

We acknowledge the help with data collection and administrative support by Cees Haaring (UMCU), Karin Flobbe (AZM), Mirjam Borg (VUMC), Pascal Verzijl (Antonius), Jan Wolfers (AMC), Peter van Ooijen (UMCG), Willie Donkers (Elkerliek), and Robert Dragt (Gelre).

Funding This study was funded by a program grant from the Netherlands Organization for Scientific Research-Medical Sciences (NWO-MW) grant 40-00812-98-07-005). The funders had no involvement in the study design, data collection, analysis and interpretation, the writing of the report, or the decision to submit the paper for publication.

Open Access This article is distributed under the terms of the Creative Commons Attribution Noncommercial License which permits any noncommercial use, distribution, and reproduction in any medium, provided the original author(s) and source are credited. 


\section{Appendix}

Patients who experienced a fatal or non-fatal cardiovascular event specified according the ICD codes

\begin{tabular}{lll}
\hline Disease & Codes & Number (fatal) \\
\hline Ischemic heart disease & $410-414$ & $273(84)$ \\
peripheral arterial disease (PAOD) & $440,443,444$ & $40(14)$ \\
aortic aneurysm & 441 & $19(15)$ \\
cerebrovascular disease & $430-438$ & $59(27)$ \\
non-rheumatic valve disease & 424 & $43(19)$ \\
Other forms of Heart disease (e.g. dysrythmias, heart failure, cardiomyopathy) & $425,427,428$ & $81(79)$ \\
Total & & $515(238)$ \\
\hline
\end{tabular}

Please note that in case of multiple valid endpoints in the same patient, cause of death prevailed over hospital admissions or else the first hospital discharge diagnosis was used.

\section{References}

1. Horton KM, Post WS et al (2002) Prevalence of significant noncardiac findings on electron-beam computed tomography coronary artery calcium screening examinations. Circulation 5:532-534

2. Jacobs PC, Mali WP et al (2008) Prevalence of incidental findings in computed tomographic screening of the chest: a systematic review. J Comput Assist Tomogr 2:214-221

3. Truong MT, Munden RF (2003) Lung cancer screening. Curr Oncol Rep 4:309-312

4. Wisnivesky JP, Mushlin AI et al (2003) The cost-effectiveness of low-dose CT screening for lung cancer: preliminary results of baseline screening. Chest 2:614-621

5. Song ZZ, Ma J (2007) Incidental detection of aortic valve calcification at CT. Radiology 2:611-612

6. Allison MA, Cheung P et al (2006) Mitral and aortic annular calcification are highly associated with systemic calcified atherosclerosis. Circulation 6:861-866

7. Agmon Y, Khandheria BK et al (2001) Aortic valve sclerosis and aortic atherosclerosis: different manifestations of the same disease? Insights from a population-based study. J Am Coll Cardiol 3:827-834

8. Otto CM, Lind BK et al (1999) Association of aortic-valve sclerosis with cardiovascular mortality and morbidity in the elderly. N Engl J Med 3:142-147

9. Volzke H, Haring R et al (2010) Heart valve sclerosis predicts allcause and cardiovascular mortality. Atherosclerosis 2:606-610

10. Fox CS, Vasan RS et al (2003) Mitral annular calcification predicts cardiovascular morbidity and mortality: the Framingham Heart Study. Circulation 11:1492-1496

11. De BG, Ambrosioni E et al (2004) European guidelines on cardiovascular disease prevention in clinical practice. Third Joint Task Force of European and other Societies on Cardiovascular Disease Prevention in Clinical Practice (constituted by representatives of eight societies and by invited experts). Atherosclerosis 2:381-391

12. Witteman JC, Kok FJ et al (1986) Aortic calcification as a predictor of cardiovascular mortality. Lancet 8516:1120-1122

13. Eisen A, Tenenbaum A et al (2008) Calcification of the thoracic aorta as detected by spiral computed tomography among stable angina pectoris patients: association with cardiovascular events and death. Circulation 13:1328-1334
14. Iribarren C, Sidney S et al (2000) Calcification of the aortic arch: risk factors and association with coronary heart disease, stroke, and peripheral vascular disease. JAMA 21:2810-2815

15. Arad Y, Goodman KJ et al (2005) Coronary calcification, coronary disease risk factors, C-reactive protein, and atherosclerotic cardiovascular disease events: the St. Francis Heart Study. J Am Coll Cardiol 1:158-165

16. Kondos GT, Hoff JA et al (2003) Electron-beam tomography coronary artery calcium and cardiac events: a 37-month follow-up of 5635 initially asymptomatic low- to intermediate-risk adults. Circulation 20:2571-2576

17. Prentice RL (1986) A case-cohort design for epidemiologic cohort studies and disease prevention trials. Biometrika 73:1-11

18. Onland-Moret NC, van der A DL et al (2007) Analysis of casecohort data: a comparison of different methods. J Clin Epidemiol $4: 350-355$

19. Koos R, Kuhl HP et al (2006) Prevalence and clinical importance of aortic valve calcification detected incidentally on CT scans: comparison with echocardiography. Radiology 1:76-82

20. Mahnken AH, Muhlenbruch G et al (2007) MDCT detection of mitral valve calcification: prevalence and clinical relevance compared with echocardiography. AJR Am J Roentgenol 5:1264-1269

21. Shemesh J, Henschke CI et al (2006) Frequency of coronary artery calcification on low-dose computed tomography screening for lung cancer. Clin Imaging 3:181-185

22. Jacobs PC, Prokop M et al (2010) Semiquantitative assessment of cardiovascular disease markers in multislice computed tomography of the chest: interobserver and intraobserver agreements. J Comput Assist Tomogr 2:279-284

23. De Bruin A, Kardaun JW et al (2004) Record linkage of hospital discharge register with population register: experiences at Statistics Netherlands. Stat J UN Econ Comm Eur 21:23-32

24. Herings RM, Bakker A et al (1992) Pharmaco-morbidity linkage: a feasibility study comparing morbidity in two pharmacy based exposure cohorts. J Epidemiol Community Health 2:136-140

25. Paas GR, Veenhuizen KC (2002) [Research on the validity of the LMR]. Utrecht, Prismant

26. Reitsma JB, Kardaun JW et al (2003) Possibilities for anonymous follow-up studies of patients in Dutch national medical registrations using the Municipal Population Register: a pilot study. Ned Tijdschr Geneeskd 46:2286-2290

27. World Health Organization International Classes of Disease (2000) World Health Organization, Geneva. Available via http:// 
apps.who.int/classifications/apps/icd/icd10online/. Accessed 10 March 2010

28. Mackenbach JP, Van Duyne WM et al (1987) Certification and coding of two underlying causes of death in The Netherlands and other countries of the European Community. J Epidemiol Community Health 2:156-160

29. LaMonte MJ, FitzGerald SJ et al (2005) Coronary artery calcium score and coronary heart disease events in a large cohort of asymptomatic men and women. Am J Epidemiol 5:421-429

30. Itani Y, Watanabe S et al (2006) Relationship between aortic calcification and stroke in a mass screening program using a mobile helical computed tomography unit. Circ J 6:733-736

31. Brenner DJ, Hall EJ (2007) Computed tomography-an increasing source of radiation exposure. N Engl J Med 22:2277-2284

32. Messika-Zeitoun D, Aubry MC et al (2004) Evaluation and clinical implications of aortic valve calcification measured by electron-beam computed tomography. Circulation 3:356-362

33. Lloyd-Jones D, Adams R et al (2009) Heart disease and stroke statistics-2009 update: a report from the American Heart
Association Statistics Committee and Stroke Statistics Subcommittee. Circulation 3:480-486

34. Katz R, Budoff MJ et al (2009) Relationship of metabolic syndrome with incident aortic valve calcium and aortic valve calcium progression: the Multi-Ethnic Study of Atherosclerosis (MESA). Diabetes 4:813-819

35. Owens DS, Katz R et al (2008) Interaction of age with lipoproteins as predictors of aortic valve calcification in the multi-ethnic study of atherosclerosis. Arch Intern Med 11:1200-1207

36. Ix JH, Shlipak MG et al (2007) Kidney function and aortic valve and mitral annular calcification in the Multi-Ethnic Study of Atherosclerosis (MESA). Am J Kidney Dis 3:412-420

37. Katz R, Wong ND et al (2006) Features of the metabolic syndrome and diabetes mellitus as predictors of aortic valve calcification in the Multi-Ethnic Study of Atherosclerosis. Circulation 17:2113-2119

38. Nasir K, Katz R et al (2008) Ethnic differences between extracoronary measures on cardiac computed tomography: multi-ethnic study of atherosclerosis (MESA). Atherosclerosis 1:104-114 